\title{
Testing and optimizing the spectral response of digital cameras
}

Document Version

Accepted author manuscript

Link to publication record in Manchester Research Explorer

\section{Citation for published version (APA):}

Oulton, D., \& Yu, D. K. C. (2005). Testing and optimizing the spectral response of digital cameras. Imaging Science Journal, 53(1), 1-11.

\section{Published in:}

Imaging Science Journal

\section{Citing this paper}

Please note that where the full-text provided on Manchester Research Explorer is the Author Accepted Manuscript or Proof version this may differ from the final Published version. If citing, it is advised that you check and use the publisher's definitive version.

\section{General rights}

Copyright and moral rights for the publications made accessible in the Research Explorer are retained by the authors and/or other copyright owners and it is a condition of accessing publications that users recognise and abide by the legal requirements associated with these rights.

\section{Takedown policy}

If you believe that this document breaches copyright please refer to the University of Manchester's Takedown Procedures [http://man.ac.uk/04Y6Bo] or contact uml.scholarlycommunications@manchester.ac.uk providing relevant details, so we can investigate your claim.

\section{OPEN ACCESS}




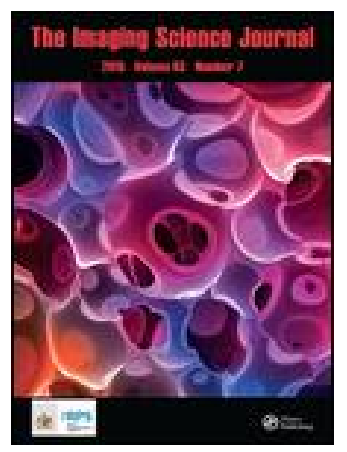

The Imaging Science Journal

\section{Testing and optimizing the spectral response of digital cameras}

\section{P Oulton \& D K C Yu}

To cite this article: D P Oulton \& D K C Yu (2005) Testing and optimizing the spectral response of digital cameras, The Imaging Science Journal, 53:1, 1-11, DOI: 10.1179/136821904X24091

To link to this article: https://doi.org/10.1179/136821904X24091

$$
\text { 曲 Published online: } 29 \text { Nov } 2013 .
$$

Submit your article to this journal

LII Article views: 12

Q View related articles $₫$ 


\title{
Testing and optimizing the spectral response of digital cameras
}

\author{
D P Oulton* and D K C Yu \\ Department of Textiles, UMIST, Sackville Street, Manchester M60 1QD, UK
}

\begin{abstract}
A colorimetric analysis and design method for cameras is presented which enables a camera to be used as an imaging colorimeter, capable of capturing an accurate record of the Commission Internationale de l'Éclairage (CIE) coordinates of the imaged objects. The spectral response characteristics of some of the component observers in the 1964 CIE Standard Observer average are first analysed and compared. CIE colour coordinates are calculated using both the Standard Observer average and individual observer CMFs for each of 32 test colours. The personal coordinates for each observer are then linked to differences in their visual response CMFs at the spectral level. A colorimetric testing and optimization method for cameras is then reported, which allows the colour capture properties of imaging systems to be analysed, controlled and modified. The method is based on imaging spectrally defined colour charts and is used to demonstrate that even minor differences in the chosen RGB filter/sensor characteristics substantially affect the numeric accuracy of the colour-defining information in images. Colour imaging error is quantified and minimized, first by establishing the camera RGB to CIE XYZ relationship, and then by adjusting the spectral response of the sensors. Under controlled lighting and exposure conditions, the design method enables output pixel-colour definitions that are a close analogue of the measured CIE XYZ tristimulus values for the imaged surface colours.
\end{abstract}

Keywords: spectral response, digital camera, colorimetric analysis, CIE

\section{INTRODUCTION}

Digital camera users are usually familiar with image colour adjustment based on the control of exposure and lighting, and on device profiling and digital photoprocessing. Such methods do not, however, correct the colorimetric detail in the image. As a result, certain colours are often poorly represented and appear incorrect in hue or chroma relative to other colours in the reproduced image.

The reported investigation concerned the design of a colorimetrically accurate camera for use as an imaging colorimeter, capable of characterizing colour changes in food and food products. To achieve this objective, it was found necessary to re-design the detailed spectral response characteristics of the camera. The outcome is

The MS was accepted for publication on 17 December 2004.

* Corresponding author: Senior Research Fellow, Department of Textiles, UMIST, Sackville Street, Manchester UK M60 1QD, UK; e-mail: david.oulton@textiles.umist.ac.uk a diagnostic test and optimization method, by which the colorimetric design of imaging systems can be reengineered to specific requirements.

The adopted methodology is similar to that proposed by Martinez-Verdu et al., ${ }^{1}$ but it uses an improved 'grey-scale tracking' algorithm described by Oulton and Porat ${ }^{2}$ to optimize the three-dimensional colorimetric model. Spectral response optimization is then based on two alternative approaches both aimed at creating a camera whose colour matching functions (CMFs) are a close analogue of the Commission Internationale de l'Éclairage (CIE) Standard Observer response.

Spectral response optimization addresses the problem of metamerism in the camera response. Conditional (or metameric) visual matches exist between certain pairs of surface colours with dissimilar spectral reflectance curves. The existence of such conditional matches is accurately predicted by spectral summation in the CIE tristimulus sum model of the human visual response. 
Illuminant metamerism is evident when a single observer viewing a matching pair of surface-colour samples sees a mismatch between the previously matching sample pair after the illuminant is changed. The breakdown of a conditional colour match on change of illuminant is discussed more fully by Luo et $a l^{3}$ It is caused by differences in colour constancy between the members of the sample pair being matched under change of illuminant. If both members change colour by the same amount, in the same direction, the match is maintained under the illuminant change.

Spectral response metamerism causes breakdown of conditional matches on 'change of observer', while keeping the illuminant constant. Thus, individual human observers (or indeed a camera with particular CMFs) may disagree with the CIE Standard Observer over the existence of a particular metameric match. All reproduced images are inevitably only a metameric match to the imaged objects, and it is spectral response metamerism that is responsible for the anomalous appearance of certain colours in images.

In the well-known reference work Billmeyer and Saltzman's Principles of Color Technology, Roy Berns ${ }^{4}$ describes the use of a 'standard deviate observer' to characterize this phenomenon. The present paper uses an alternative method proposed by Oulton and Taylor, ${ }^{5}$ based on a representative set of measured reflectance curves. CIE coordinates of the test samples are calculated using the Standard Observer CMFs and a set of camera CMFs, respectively, and the resulting colour difference definitions can then be analysed to reveal the spectral-response effects produced by different sensor characteristics.

In the following sections, the spectral response of the CIE Standard Observer and that of digital cameras are compared and analysed, and this leads to a colorimetric design method that can be used to minimize spectral response metamerism.

All quoted CIE colour specifications are specified using the ASTM E 308-95 standard calculation method for the CIE $196410^{\circ}$ Supplementary Standard Observer. ${ }^{6}$

\section{TEST COLOURS USED TO DETECT AND QUANTIFY SPECTRAL-RESPONSE METAMERISM}

Two sets of test colours were used to quantify the effects of spectral response differences. One has 32 samples, defined by the measured reflectance curves of samples from the NCS colour atlas. ${ }^{7}$ The second set consists of synthetic reflectance curve definitions for 20 samples of textile yarn. Four combinations of high and low lightness with high and low chroma, respectively, in eight or five hue groups are used. They are distributed over a large volume of colour space, giving either 32 or 20 test colours.

The 32-sample set was used to analyse the differences in human observer CMFs. The synthetic spectral data of the 20-sample set were used to analyse the effects of differences in spectral sensitivity of cameras, because they have better colour constancy on change of illuminant. For practical field use in camera calibration, the Macbeth Color Checker ${ }^{8}$ and the Kodak Grey Scale, ${ }^{9}$ imaged under a simulated $\mathrm{D}_{65}$ Illuminant, are a useful alternative to the above research test sets.

The effects of change in spectral response are quantified using the change in calculated colour specification for the members of the relevant test set, expressed as a size and direction of colour difference delta $E$. The chosen measure is the calculated colour difference using the CIE $196410^{\circ}$ Observer and CMC $(2: 1)$ definition given by Berns. ${ }^{10}$

\section{METAMERIC DEVIATIONS IN THE RESPONSE OF HUMAN OBSERVERS}

A change of human observer is shown by Oulton and Taylor ${ }^{5}$ to produce significant differences in perceived colour specification. The size and direction of change are described by Oulton and Taylor ${ }^{5}$ and quoted below.

Oulton and Taylor used the CMFs of 20 individual observers measured by Stiles and Burch. ${ }^{11}$ The observers were component members the 1964 CIE Supplementary Standard Observer average, tabulated in detail by Wyszecki and Stiles. ${ }^{12}$

CIE colour coordinates were calculated using the relevant observer CMFs for each of the 32 measured reflectance curves in the test set. The CMC $(2: 1)$ colour differences under standard illuminant $\mathrm{D}_{65}$ were then calculated relative to the Standard Observer CMF definitions. A total of 640 calculations of colour specification alteration on change of observer were made.

\subsection{Human observer results}

The altered perception of colour specifications on change of observer is quantified in Table 1. Virtually 
all the colours are perceived differently by the individual observers, but metameric disagreement only occurs when specific hue groups interact differently with the changed CMFs. Thus, Table 1 gives the individual hue-group and mean delta $E$ values obtained for each of the test observers.

The relative colour definition in a given scene would not be affected if the change in spectral response produced an identical change in colour specification for all the imaged objects. In practice, however, each individual spectral response gives a distinct set of colour coordinates which distribute the test colours differently in colour space, according to their metameric properties.

The size of the quoted delta $E$ values may be interpreted by reference to the visual matching experiments of $\mathrm{Yu}$ and Oulton, ${ }^{13}$ where object to image visual colour differences are quantified in terms of CMC (2:1) delta $E$. They found that a measured delta $E \leqslant 1$ was typically reported as an acceptable colour match, and any difference delta $E \geqslant 3$ was regarded as a poor match.

In the current data set, the mean colour specification change for all the colours in the test set and all the observers is delta $E=0.97 \mathrm{CMC}(2: 1)$. However, six observers out of the 20 have a mean delta $E>1$ across the set of colours, and the individual observer maximum in Table 1 (delta $E=8.14$ ) represents a substantial perceived colour difference. There is thus significant potential for metameric failure of colour definition on change of human observer.

The detailed metameric hue, and chroma differences are best illustrated and related to cameras using a synthesized camera response rather than observer CMFs. Figure 1, illustrates the CIE $a^{*} b^{*}$ colour coordinate differences that relate the Standard Observer response to the camera response.

In Fig. 1, the metameric difference between the two spectral responses clearly affects each colour group differently. For example, the reds and greens move in opposite directions in colour space, because they both have enhanced chroma in the camera response relative to that of the Standard Observer. Also, the yellows are a little redder and brighter, the magentas are a little bluer, and the cyans are significantly greener. It would thus be impossible to correct all the individual colours by adjusting the individual RGB channel outputs. The CMC LCH colour differences for each colour group are given in Table 2.

The above synthesized example of spectral response metamerism is comparable (in severity and metameric effects) with that found in some high-quality digital cameras. There may indeed be good reason to create such a response, for example to make the resulting

Table 1 Detailed variation in colour-identity specification for 20 individual observers, quantified as CMC delta $E$ values relative to the calculated colour specification for CIE 1964 10o Supplementary Standard Observer

\begin{tabular}{|c|c|c|c|c|c|c|c|c|c|c|}
\hline \multirow[t]{2}{*}{ Colour group } & \multicolumn{10}{|c|}{ Actual Observers (Set 1) } \\
\hline & WSS1 & JMB2 & EMK3 & GEVL4 & MCB5 & DLT6 & EML7 & MHP8 & EA9 & RNW10 \\
\hline$\overline{\text { Blue }}$ & 0.47 & 0.75 & 1.72 & 2.64 & 1.03 & 0.57 & 0.44 & 1.09 & 0.65 & 1.91 \\
\hline Blue-Red & 0.44 & 0.51 & 0.43 & 0.65 & 0.38 & 0.25 & 0.20 & 0.40 & 0.48 & 0.12 \\
\hline Red & 0.71 & 0.63 & 0.98 & 0.57 & 0.33 & 0.29 & 0.33 & 0.21 & 1.02 & 1.13 \\
\hline Red-Yellow & 0.93 & 0.63 & 1.49 & 1.32 & 0.44 & 0.30 & 0.35 & 0.51 & 0.96 & 2.35 \\
\hline Yellow & 0.45 & 0.80 & 0.78 & 1.89 & 0.79 & 0.58 & 0.39 & 0.98 & 0.61 & 1.52 \\
\hline Yellow-Green & 0.57 & 0.76 & 0.28 & 1.27 & 0.57 & 0.40 & 0.31 & 0.74 & 0.68 & 0.54 \\
\hline Green & 0.33 & 0.67 & 0.81 & 0.40 & 0.22 & 0.08 & 0.09 & 0.19 & 0.50 & 0.63 \\
\hline Green-Blue & 0.22 & 0.68 & 1.22 & 1.00 & 0.42 & 0.17 & 0.20 & 0.32 & 0.56 & 1.11 \\
\hline Overall mean & 0.52 & 0.68 & 0.96 & 1.22 & 0.52 & 0.33 & 0.29 & 0.55 & 0.68 & 1.16 \\
\hline \multirow[t]{2}{*}{ Colour group } & \multicolumn{10}{|c|}{ Actual Observers (Set 2) } \\
\hline & MG11 & PSW12 & FJC13 & JAR 14 & W15 & JSP16 & WDW17 & JB18 & GW19 & DBJ20 \\
\hline Blue & 1.55 & 0.99 & 1.46 & 3.58 & 2.95 & 4.13 & 0.87 & 0.80 & 1.27 & 4.55 \\
\hline Blue-Red & 0.21 & 0.26 & 0.43 & 0.85 & 0.54 & 1.29 & 0.33 & 0.16 & 0.13 & 0.64 \\
\hline Red & 0.22 & 0.40 & 0.38 & 0.46 & 0.88 & 2.62 & 0.48 & 0.64 & 0.92 & 0.83 \\
\hline Red-Yellow & 0.90 & 0.21 & 0.49 & 2.90 & 3.15 & 8.14 & 0.42 & 0.88 & 1.70 & 3.43 \\
\hline Yellow & 1.01 & 0.46 & 0.87 & 3.00 & 2.86 & 2.20 & 0.86 & 0.57 & 1.08 & 3.04 \\
\hline Yellow-Green & 0.55 & 0.35 & 0.68 & 1.67 & 1.52 & 0.74 & 0.64 & 0.26 & 0.38 & 1.55 \\
\hline Green & 0.46 & 0.24 & 0.25 & 0.50 & 0.40 & 2.59 & 0.19 & 0.28 & 0.36 & 1.14 \\
\hline Green-Blue & 0.80 & 0.45 & 0.54 & 1.52 & 1.27 & 2.90 & 0.39 & 0.51 & 0.73 & 2.30 \\
\hline Overall mean & 0.71 & 0.42 & 0.64 & 1.81 & 1.70 & 3.07 & 0.52 & 0.51 & 0.82 & 2.19 \\
\hline
\end{tabular}




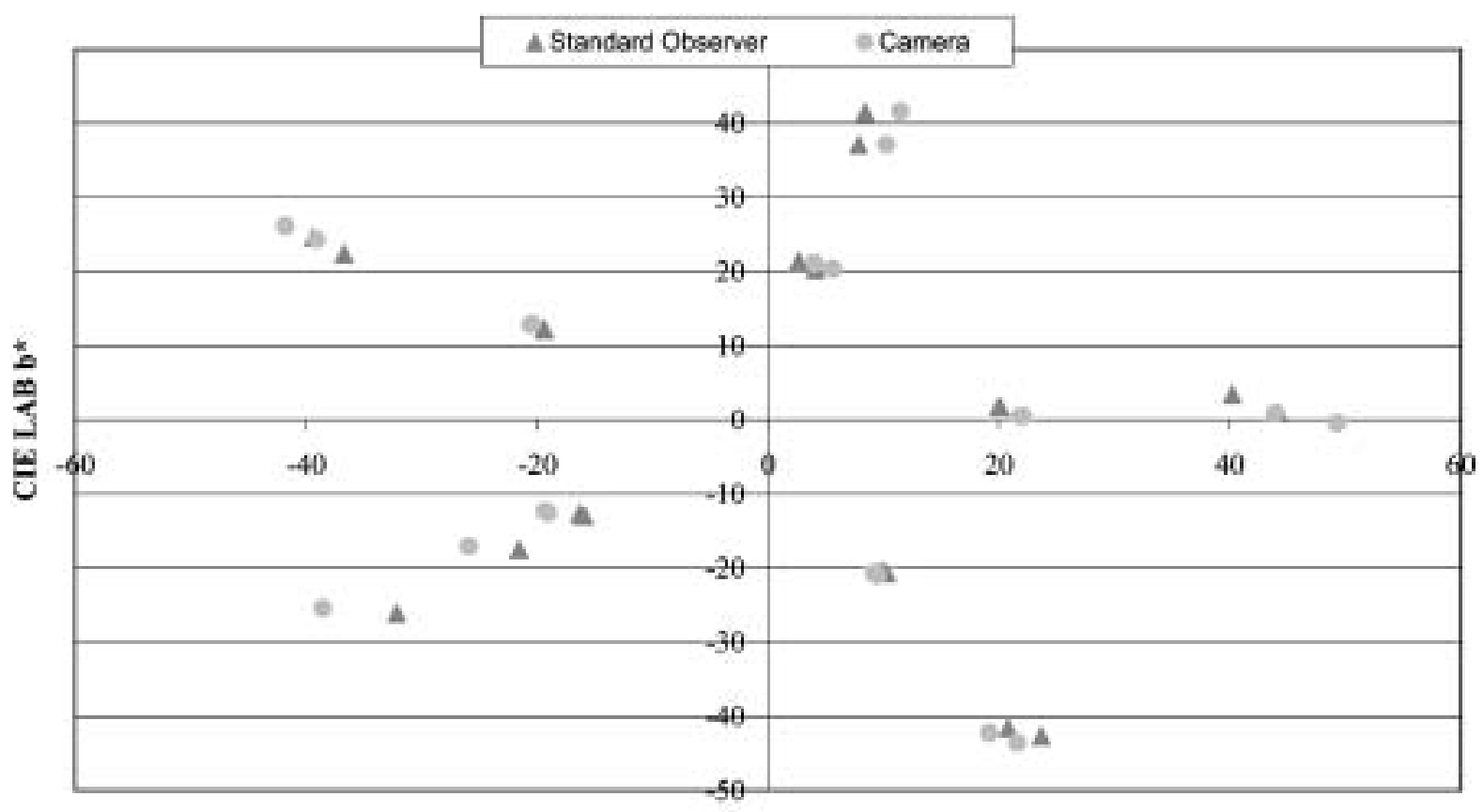

CIE LAB a *

1 CIE a*b* (1964 10 Supplementary Standard Observer) coordinates for set of 20 test colours versus equivalent camera coordinates

pictures visually pleasing. Also, in some digital cameras the spectral response may be adjusted to improve the blue channel output, or perhaps to emphasize certain colours. Whatever the motivation, the result is a very different spectral response characteristic from that of the CIE Standard Observer, and some of the commercially available cameras tested in the project were typically found to generate CMC delta $E$ maxima in excess of ten colour difference units for the chosen test set (see $\mathrm{Yu}$ and Oulton ${ }^{13}$ ).

\section{SPECTRAL RESPONSE METAMERISM AND COLORIMETRIC DESIGN OF CAMERAS}

In the following sections, a computer model is described which allows the colorimetric design of

Table 2 Camera versus Standard Observer colour coordinate differences (averaged by colour group)

\begin{tabular}{lrllll}
\hline & \multicolumn{5}{l}{ CMC (2:1) colour difference units } \\
\cline { 2 - 6 } & Red & Yellow & Green & Cyan & Magenta \\
\hline Delta L & -0.107 & 0.002 & 0.094 & 0.032 & -0.127 \\
Delta C & 1.254 & 0.333 & 1.134 & 1.259 & -0.033 \\
Delta H & 1.371 & 1.489 & 0.729 & 2.559 & 0.930 \\
\hline
\end{tabular}

digital cameras to be analysed and optimized. The process involves two distinct optimizations, for the three-channel colorimetric error and the $n$-channel spectral-response error, respectively. Once overall three channel error has been minimized, the residual delta $E$ colour differences originate mainly at the spectral level and quantify the effects of spectralresponse metamerism.

\subsection{Methodology}

All automatic compensation features, such as that for the incident illuminant, are first turned off and set to a constant reference value, and all RGB values are then determined at a reference shutter speed, aperture and light intensity.

The presented method is known to broadcast television engineers as 'grey-scale tracking', because it uses an imaged grey scale to calibrate the camera characteristics. A scalable linear model of the digital RGB camera output values is required, such that the camera output values (denoted $R^{\prime} G^{\prime} B^{\prime}$ ) are expressed in the same Trichromatic Units (or T-Units) in which CIE XYZ Standard Observer tristimulus values are expressed. If this unit transformation is correctly determined, the CIE $X Y Z$ tristimulus values of an imaged object are then a strictly constant matrix 
product of the camera $R^{\prime} G^{\prime} B^{\prime}$ values. Unit definition by grey-scale tracking has a fundamental basis in colorimetric theory, because the spectral tristimulus values that define the CIE Standard Observer CMFs are normalized such that they have coordinates $X=Y=Z$ by reference a light source with equal energy at all wavelengths (Illuminant $S_{\mathrm{E}}$ ). This normalization defines the axis of visual neutrality in the CIE system to which all other $X, Y$ and $Z$ values are referenced numerically.

In the CIE system, the $Y$ value carries all the visual intensity information, and $Y$ therefore also specifies the T-Unit scaling in the $X$ and $Z$ dimensions of the tristimulus sum. It follows that a correct definition of camera-specific T-Units can be established by creating an equivalent neutral reference axis $R^{\prime}=G^{\prime}=B^{\prime}$. If the camera exactly reproduces (or tracks) the grey scale, its $R^{\prime}=G^{\prime}=B^{\prime}$ axis is then offset by a constant ratio from the CIE visual neutrality axis $X=Y=Z$. The relevant $R^{\prime} G^{\prime} B^{\prime}$ offset is the product of the measured CIE $x, y, z$ chromaticity of a reference target white under the chosen illuminant, and its measured camera $\mathrm{R}: \mathrm{G}: \mathrm{B}$ ratio. The axis offset is dependent on the chosen illuminant.

In effect, grey-scale tracking creates the linear model by isolating and quantifying the non-linear $\mathrm{T}$-Unit characteristic of the camera, such that $R^{\prime}=f(\mathrm{R})$, $G^{\prime}=f(\mathrm{G})$ and $B^{\prime}=f(\mathrm{~B})$, and $R^{\prime}=G^{\prime}=B^{\prime}$ at each grey level. Critically, the three functional products $R^{\prime} G^{\prime} B^{\prime}$ are thereby exactly balanced and linearized numerically relative to $X, Y$ and $Z$ at all digital output levels. This is a refinement of the process described by Berns ${ }^{14}$ as 'finding a scalable function' and 'finding the linear model'. The use of the T-Unit concept in the CIE system is elaborated in more detail by Oulton ${ }^{2,15}$ and by Sproson ${ }^{16}$ in connection with cameras.

\subsubsection{Unit definition}

An appropriate camera-response white point is first determined under a standard illuminant using a good-quality white reference target, such that one or more of the output $\mathrm{R}, \mathrm{G}$ or B values is at or near maximum. The resulting $R^{\prime} G^{\prime} B^{\prime}$ values are then scaled relative to imaged object CIE $Y=0$ to $100+$ (thus allowing for the specification fluorescent colours). The dark current (i.e. zero light) RGB values should be checked, and an offset zero point on the CIE $Y$ scale should be established if necessary.

At each point on the grey scale, the measured CIE $x, y, Y$ coordinates of an imaged grey patch are related to camera RGB values in a six-column lookup table (LUT) ordered on $Y$. Typically, the RGB values will then be smoothly non-linear relative to CIE $Y$. If the grey-scale tracking is incorrect, the camera $\mathrm{R}: \mathrm{G}: \mathrm{B}$ ratios will also deviate from the white point ratio (after allowing for any variations in the target grey $x, y, z$ chromaticities). Any such deviations from either linearity or neutral balance ratio must now be calibrated in order to create the T-Unit definition of the camera output.

The required scalable T-Unit basis for the linear $R^{\prime} G^{\prime} B^{\prime}$ model is derived from the LUT by non-linear interpolation, preferably using spline functions. Three sets of function-defining parameters are optimized simultaneously by minimizing the total delta $E$ between the CIE $x, y, Y$ coordinates of the grey-scale target patches and the equivalent camera output values. Balancing equates camera channel output three-dimensionally at all levels, scaling equates the output $Y$ values, and flexible linearization replaces the conventional gamma curve approximation such that the three individual RGB nonlinearities of the camera response are accurately determined relative to CIE $Y$.

Scaling, balance and linearity are typically unstable over time, and differ from camera to camera. They thus require current-state and device-specific recalibration for optimum performance. After each (single or multiple exposure) grey-scale calibration, the three functions establish an accurate and strictly uniform definition of T-Unit camera response common to all three channels and all output levels. The result is a comprehensive calibration of the camera that is significantly more accurate than that provided by conventional white point and gamma curve device characterization.

\subsubsection{Unique linear cross-dependency}

The three spectrally integrated channel responses generated by grey-scale tracking now allow a nominally constant $3: 3$ matrix of $\mathrm{RGB} / \mathrm{XYZ}$ crossdependency coefficients to be optimized (The matrix coefficients are only a strict constant of the camera characteristic if spectral metamerism is absent.)

The optimization is by reference to the CIE $x, y, Y$ coordinates of a representative set of coloured target patches, e.g. those of the Macbeth colour chart. ${ }^{8}$ An initial value for the nine coefficients to be optimized can be calculated directly from the CIE $x, y, \mathrm{z}$ chromaticity of the individual RGB spectral responses; this is 
not essential, however. Sproson ${ }^{16}$ gives further detail of this derivation. In effect, this matrix optimization establishes the minimum possible metameric delta $E$ value for the set of test patches, and the camera then delivers a (potentially large) residual colour coordinate error that is mainly the $n$-dimensional product of the current metameric spectral response.

\subsection{Spectral optimization}

The subsequent second-stage optimization concerns the spectral response, and generates a spectral characteristic that is the closest available analogue of CIE 1964 Supplementary Standard Observer.

This second-stage computer model takes as input:

1. the set of 20 surface-colour reflectance-curve definitions, which represent test colours that vary in lightness, chroma and hue, and sample the expected colour reproduction range systematically

2. the CIE Standard Observer $X Y Z$ tristimulus values for each of the measured spectral reflectance curves

3. the spectral camera characteristics under analysis (either directly measured or modelled).

The outputs of the model include:

1. two sets of CIE $\mathrm{L}^{*} \mathrm{a}^{*} \mathrm{~b}$ coordinates for each member of the colour set, calculated using the Standard Observer and the alternative 'camera characteristic' CMF weightings, respectively

2. calculated CMC $(2: 1)$ colour difference values, between the CIE $L^{*} a^{*} b^{*}$ definitions calculated for the Standard Observer and the current camera response.

Either a measured or a synthetic spectral response can be analysed. In the proposed design method, a response characteristic is synthesized by linear combination of Gaussian distribution functions over wavelength, where each such combination is in effect an analogue of spectral response sensitivity. This avoids any complications that might arise from the detailed light transmittance characteristics of physical filter media.
Alternative physical RGB filter media are simulated in the computation by adjusting the wavelength maximum and bandwidth of the Gaussian functions. The bandwidths quoted in Table 3 are equivalent to \pm 1 standard deviation either side of the mean, and the modelled bandwidth represents just over $68 \%$ of the total response. Physical filter/photoreceptor response combinations have also been used as input, to generate similar camera-response characteristics.

The available optimization variables are the nine matrix coefficients, plus the wavelength maximum and bandwidth for each of the component Gaussian functions. Wavelength maximum and bandwidth were, however, held constant in the following analysis. The optimization criterion is the overall calculated delta $E$ for the set of spectrally defined test colours.

\section{SPECTRAL RESPONSE ANALYSIS METHOD}

In order to establish the sensitivity of the design analysis method, two distinct spectral response definitions were next compared. The first 'best characteristic' has primaries chosen to have response maxima very close to those of the CIE $\bar{x}, \bar{y}, \bar{z}$ CMFs, and to have a similar bandwidth. The second slightly modified 'poor characteristic' is altered spectrally as shown in Table 3. The wavelength and bandwidth details of the poor characteristic were chosen to reflect the differences in the human spectral response used to generate Table 1.

The resulting optimized linear matrix coefficients were as follows

$$
\begin{aligned}
& {\left[\begin{array}{l}
X \\
Y \\
Z
\end{array}\right]=\left[\begin{array}{ccc}
1 \cdot 00 & 0 \cdot 09 & 0 \cdot 225 \\
0 \cdot 55 & 1 \cdot 0 & 0 \cdot 10 \\
0 \cdot 00 & 0 \cdot 001 & 1 \cdot 00
\end{array}\right] *\left[\begin{array}{l}
R^{\prime} \\
G^{\prime} \\
B^{\prime}
\end{array}\right]} \\
& {\left[\begin{array}{l}
X \\
Y \\
Z
\end{array}\right]=\left[\begin{array}{ccc}
1 \cdot 00 & 0.002 & 0 \cdot 245 \\
0 \cdot 43 & 1 \cdot 0 & 0 \cdot 19 \\
0.00 & 0.002 & 1.00
\end{array}\right] *\left[\begin{array}{l}
R^{\prime} \\
G^{\prime} \\
B^{\prime}
\end{array}\right]}
\end{aligned}
$$

\begin{tabular}{|c|c|c|c|c|c|c|}
\hline \multirow[t]{2}{*}{ Channel } & \multicolumn{2}{|l|}{ Blue } & \multicolumn{2}{|l|}{ Green } & \multicolumn{2}{|l|}{ Red } \\
\hline & Max. at Nm & Width Nm & Max. at $\mathrm{Nm}$ & Width Nm & Max. at $\mathrm{Nm}$ & Width Nm \\
\hline Best characteristic & 452 & 42 & 543 & 64 & 597 & 64 \\
\hline Poor characteristic & 455 & 40 & 550 & 50 & 610 & 50 \\
\hline
\end{tabular}

Table 3 Overall input channel characteristics of two camera configurations tested 


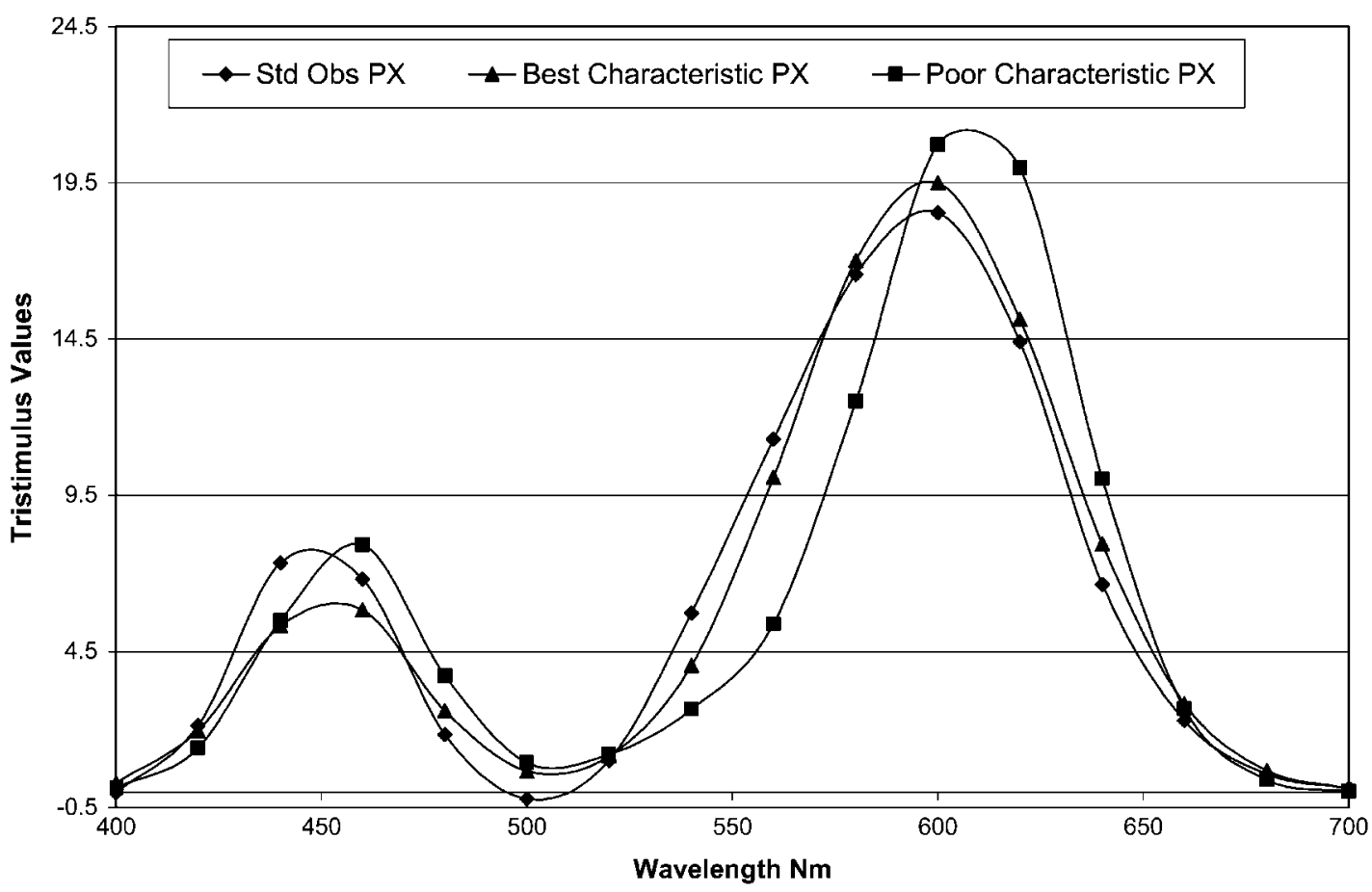

2 Spectral variation in synthesized ' $X$-Channel' response with wavelength, balanced to give equivalent tristimulus sum

The unit value diagonal of each matrix is the result of CMF normalization, and the matrices are validated for all RGB by virtue of response linearization and grey-scale tracking.

Figures 2-4 illustrate and compare the optimized spectral response of both characteristics with that of the CIE 1964 Standard Observer after three-channel balancing, linearization and scaling. All the optimizations were carried out on a spreadsheet using the Excel Solver function.

\section{TESTING THE GENERATED CAMERA CHARACTERISTICS}

A given camera design may be assumed to be a close analogue of the CIE Standard Observer if there is minimal calculated CMC $(2: 1)$ colour difference across a representative set of test colours after the three-channel optimization step. The test set should represent a good range of hues at different chroma and lightness levels and have typical surface-colour reflectance curves. The Macbeth 'Color Checker' chart $^{8}$ is a good example, because it includes samples with appropriate reflectance curves for both skin tone and foliage colours.
The measured colour-difference values in Fig. 5 represent the difference between the Standard Observer weighted tristimulus sum and the equivalent tristimulus sum calculated using the weighting provided by each of the modelled sensor characteristics.

The delta $E$ deviations in Fig. 5 represent the irreducible minimum of colour coordinate error caused by the two tested colorimetric camera designs. The errors are relatively small in the blue part of the spectrum for both the tested characteristics. This is due to the relatively small variation in the synthesized $Z$-channel response (see Fig. 4), generated by moving the response maximum by $3 \mathrm{Nm}$, and reducing the bandwidth by $2 \mathrm{Nm}$. However, the change in wavelength maximum of the Blue channel by $3 \mathrm{Nm}$ also affects the $X$ and $Y$ output channels by crossdependency (see equation 1). This adds significantly to the delta $E$ deviations of the remaining members in the test set.

Somewhat larger deviations were introduced in response maxima and bandwidth in the $R$ and $G$ responses (see Table 3) and their effect on the $X$ and $Y$ channel responses is correspondingly larger (as shown in Figs 2 and 3). This in turn produces a greater metameric effect (see Fig. 5) principally across the four lightness and chroma combinations 


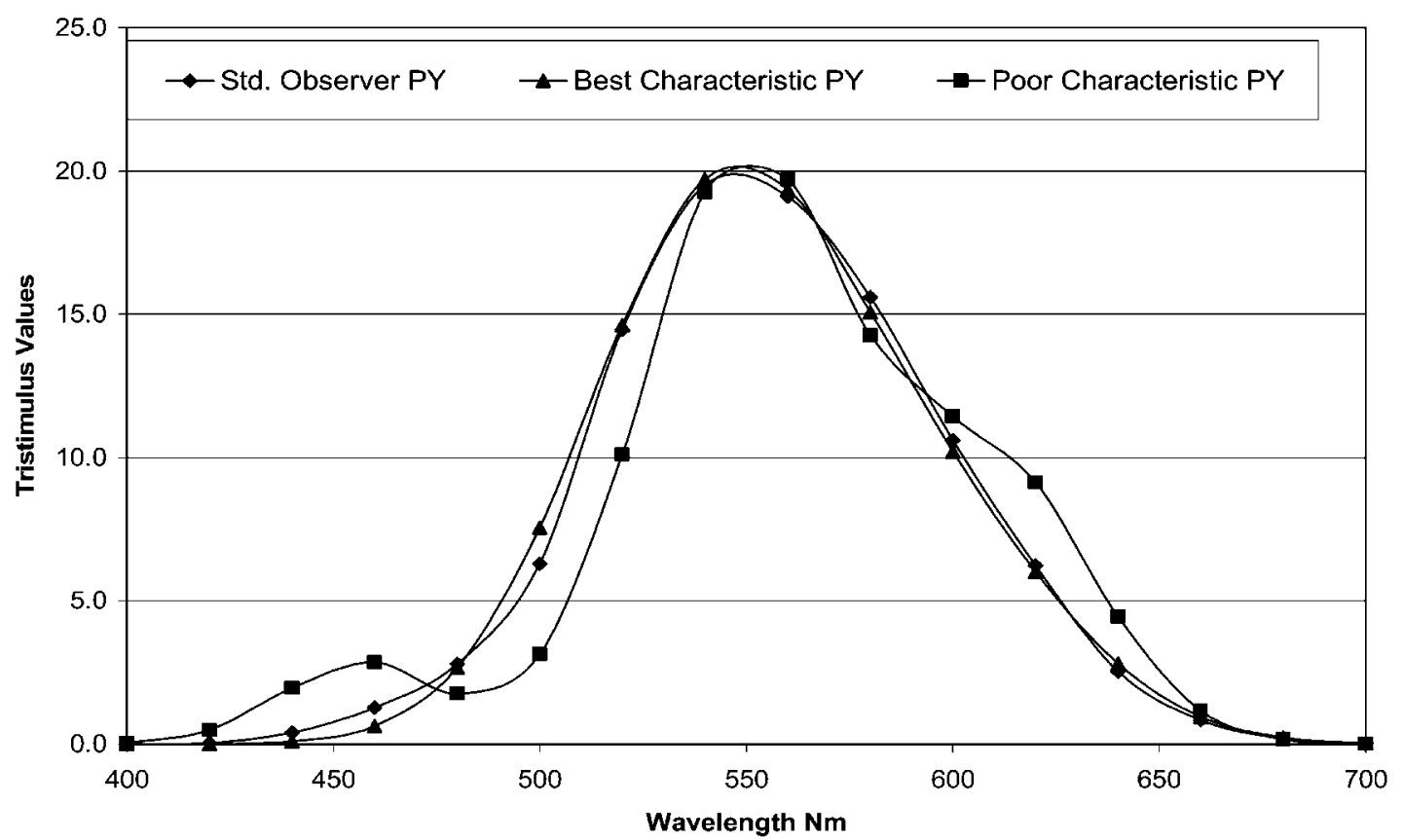

3 Spectral variation in synthesized ' $Y$-Channel' response with wavelength balanced to give equivalent tristimulus sum

in the red hue group, but also to a smaller extent, in the yellows, greens and cyans.

Table 4 shows the overall mean and the maximum residual metameric deviation from the Standard Observer response for the 'best spectral characteristic' model compared with the 'poor spectral characteristic' model. The largest hue group average deviation (delta $E$ (CMC 2:1) 4.77), is comparable with that found in the more deviant of the tabulated human observers (see Table 1).

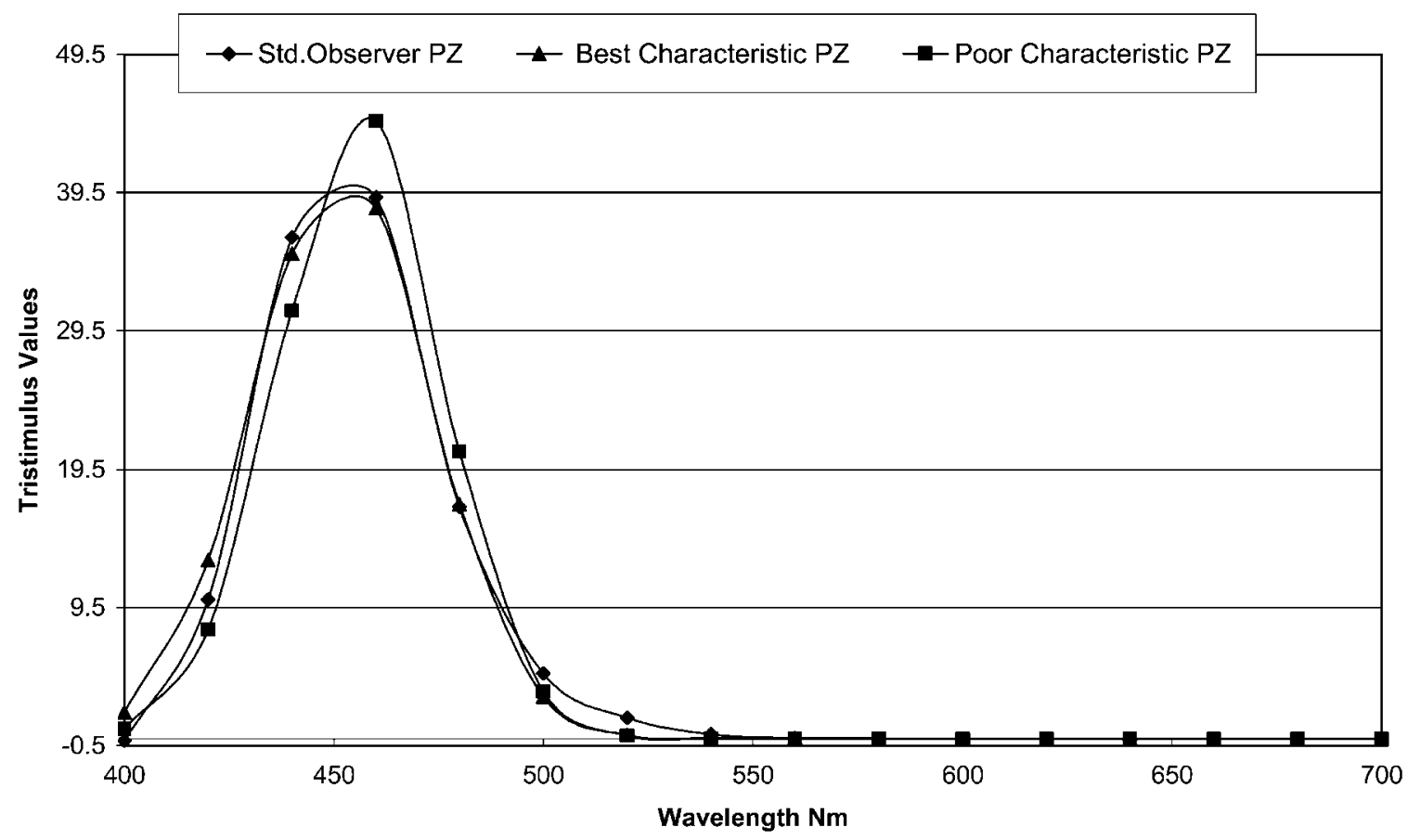

4 Variations in synthesized ' $Z$-Channel' response with wavelength, balanced to give constant tristimulus sum 


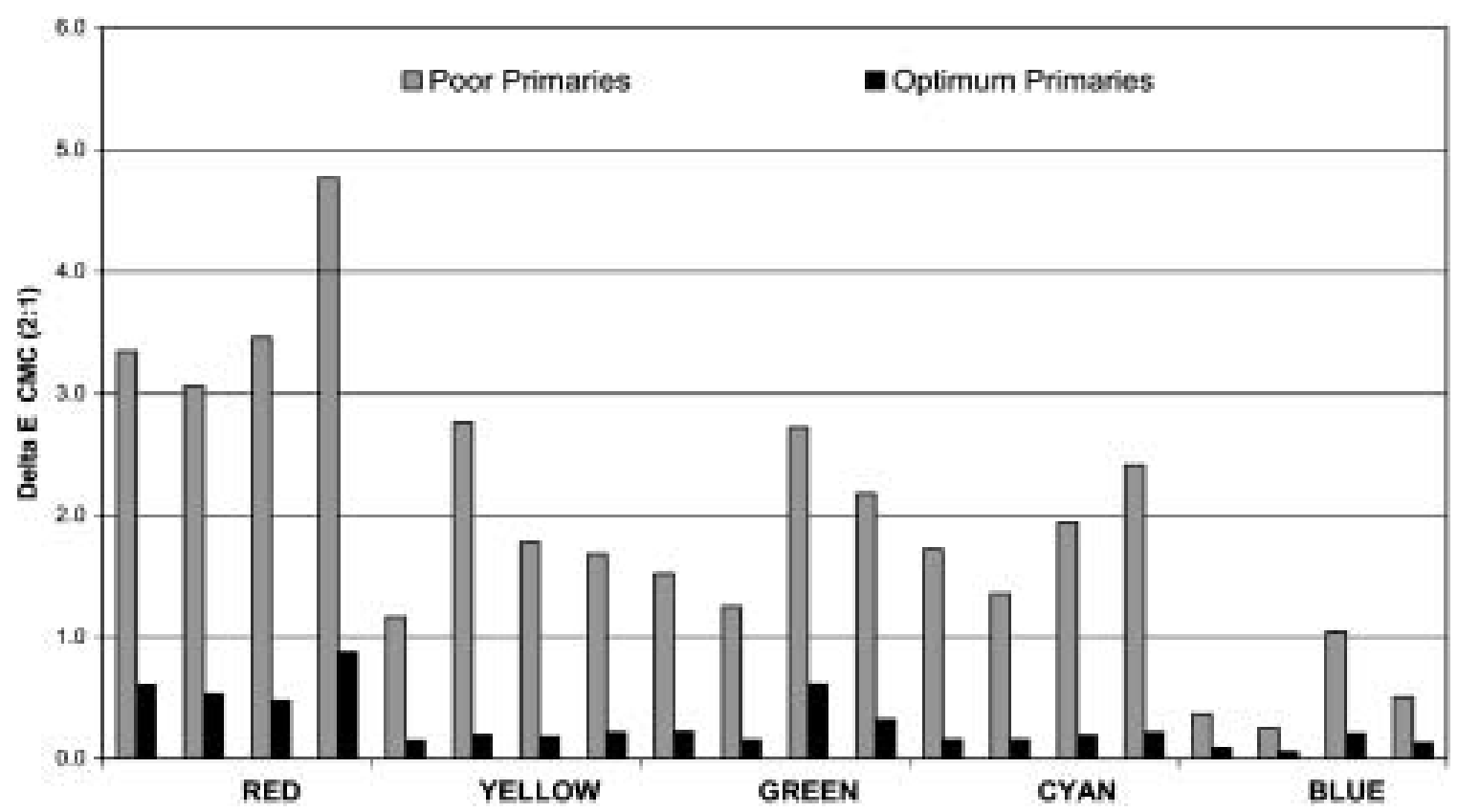

5 Detailed variation in camera colour coordinates from equivalent measured Standard Observer values; combinations of lightness and chroma are ordered left to right in each hue group as follows: Low L plus Low C, High L plus Low C, Low L plus High C, High L plus High C

The matrices quoted in equation (1) represent the optimized linear cross-dependency before and after changing the component curves as indicated in Table 2. The optimized 'best characteristics' matrix was used to generate the graphed spectral responses in Figs 2-4. The success of the optimization is confirmed by the low colorimetric errors quoted in Table 4.

The colorimetric design methodology has also been applied to real spectral response characteristics using measured photosensor responses and physical filter transmittance values. The optimization method works equally well using these data, but the resulting optimum configuration was typically a slightly less accurate analogue of the Standard Observer. Clearly, however, this is entirely dependent on the detailed distribution of filter transmittance and the photon count of the sensor over wavelength: Specific filter/ sensor combinations could, in principle, give a superior characteristic.

\section{DISCUSSION}

The optimum matrices for the two analysed camera characteristics given in equation (1) were both derived by proportionate linear combination in three dimensions, using three spectra of fixed bandwidth and wavelength maximum. It would, of course, also be possible to include bandwidth and wavelength maximum in the set of optimization variables, and the optimization would then be analogous to the practical selection of filter media and sensor characteristics.

In the current example, the spectral weighting of the 'best characteristic' is deliberately chosen so that the optimization result closely follows the weighting of the CIE Standard Observer. The analysis then demonstrates that it is only necessary to introduce relatively small changes in bandwidth and maximum wavelength in order to create a 'poor characteristic' which makes the optimization significantly less

Table 4 Mean and maximum deviation from Standard Observer colour coordinates

\begin{tabular}{lll}
\hline & Best characteristic & Poor characteristic \\
\hline Mean deviation (CMC 2:1) units & 0.29 & 1.96 \\
Maximum deviation (CMC 2: 1) units & 0.87 & 4.77 \\
\hline
\end{tabular}


successful. The resulting delta $E$ distribution is therefore clearly sensitive to the relatively minor metameric effects thus introduced.

The quoted optimization produces an equivalent (but rather uneven) spectral response in the case of the 'poor' $X$ and $Y$ responses (Figs 2 and 3). The optimized response curve is most uneven for the $Y$ channel, because the linear combination involves significant quantities of all three component curves. The result can be seen in Fig. 3. In terms of practical colour reproduction, the effect of the altered $Y$ channel response would be a selective over- and underestimation of the visual brightness of certain spectral stimuli or of broad band stimuli where these wavelengths are dominant. Likewise, the narrower bandwidth and the different peak response at longer wavelengths in the poor $X$ channel response (see Fig. 2) causes the selectively enhanced chromaticities of the red and green samples that are evident in Fig. 1.

Optimization by three-component spectral response synthesis involves filter/sensor outputs (or their Gaussian analogues), where the bandwidth of the $\mathrm{R}$ and $\mathrm{G}$ outputs overlap substantially. It is thus possible to minimize the effects of incorrect bandwidth and response maximum in the $R$ and $G$ channels. It is less easy to compensate (by crossdependent linear combination) for the effects of an incorrect blue-channel response, because of the difference in wavelength maximum and lack of overlap with the $\mathrm{R}$ and $\mathrm{G}$ sensor outputs. An incorrect response maximum in the blue channel can, however, be compensated if two distinct blue channels are used. In such a fourchannel camera design, it was demonstrated that it is possible to reduce metameric error to very low levels by appropriate choice of Gaussian components. In effect, introducing a fourth channel gives more degrees of freedom for optimizing the cross-dependency matrix between the four channel characteristics, and the synthesized $X Y Z$ output.

The number of sensors is limited to three (or at most four) in the current analysis, because the authors wish to establish a colorimetric design method for cameras and imaging colorimeters, as opposed to imaging spectrometers. The alternative imaging spectrometer approach using many narrow bandwidth filters is described in more detail by Stokman et al. ${ }^{17}$

\section{CONCLUSIONS}

A technique is presented for the colorimetric analysis and design of image capture systems at both the three channel integral-response level and the spectral level in terms of metameric effects. A suitable test method which quantifies colour recording errors is also presented. The methodology described provides an objective basis for assessing the effects of both theoretical and practical filter/sensor combinations for use in cameras, and for optimizing their colorimetric design.

The colorimetric design method seeks, first, to optimize the three-channel model by grey-scale tracking and, secondly, to achieve camera spectralresponse CMFs that are close to those of the CIE Standard Observer, thereby resolving the residual calibration error at the three-channel level. The intent is to define an objective colorimeter system which produces accurate pixel-by-pixel colour measurements. It remains to be demonstrated, however, that a camera design with a low metameric error characteristic could be implemented cost effectively in a real camera. It is also not necessarily true that such a design would produce an artistically pleasing visual appearance in terms of reproduced colour.

The intent of the spectral response analysis and optimization method is to quantify, control and modify the colorimetric design of a digital camera. Thus, alternative optimization criteria and test sets could perhaps be devised and used to engineer cameras with a wide range of visual performance characteristics. For example, the process could be used to engineer specific visually pleasing characteristics into the colorimetric design of a digital camera.

\section{ACKNOWLEDGEMENTS}

The research described arises from DTI Link Project AFM/65 'Colour Calibration for Food Appearance Measurement'. The work by Dr Pointer and Prof. Attridge at the University of Westminster during the project provided some of the background data, and also the camera system design principles used in the modelling process. Project AFM/65 also highlighted the potentially important contribution of metameric effects to image colour inaccuracy.

The authors are also indebted to Helen Taylor for the original analysis of human observer variation, ${ }^{18}$ 
and for subsequently revising the data to give results as CMC (2:1) colour differences.

\section{REFERENCES}

1 Martinez-Verdu, F. et al. J. Imag. Sci. Technol., 2003, 47(4), 279-295.

2 Oulton, D. P. and Porat, I. The control of colour by using measurement and feedback. J. Textile Inst., 1992, (3), 453.

3 Luo, M. R., Hunt, R. W. G., Rigg, B. and Smith, K. J. Recommended colour-inconstancy index. JSDC, 1999, 115(5-6), 183-188.

4 Berns, R. The standard deviate observer. In Billmeyer and Saltzman's Principles of Color Technology, 3rd edition, Ch. 2, 2000, p. 54 (Wiley Interscience, New York).

5 Oulton, D. P. and Taylor, H. Inter observer agreement on visual colour matches, and the role of metamerism as a cause of disagreement. PRA Conf. The Colour Delivery Challenge, Leeds, May 2000; in Surf. Coat. Int. $B, 2002, \mathbf{8 5}, 105-108$.

6 ASTM Standard E 308-95 'Standard Practice for Computing the Colors of Objects by Using the CIE System' (American Society for Testing and Materials, Philadelphia).

7 Hard, A., Sivik, L. and Tonnquist, G. NCS natural color system - from concepts to research and applications: part I. Color Res. Appl., 1996, 21, 180-204; part II, 206-220.
8 McCamy, C. S., Marcus, H. and Davidson, J. G. A. A color-rendition chart. J. Appl. Photogr. Eng., 1976, 2(3).

9 Kodak Grey Scale. Kodak Research and Development, Headstone Drive, Harrow HA1 1TY, UK.

10 Berns, R. CMC colour difference. In Billmeyer and Saltzman's Principles of Color Technology, 3rd edition, Ch 4D, 2000, pp. 117-118 (Wiley Interscience, New York).

11 Stiles, W. S. and Burch, J. M. N.P.L. colour-matching investigation: final report. Opt. Acta, 1959, 6, 1-26.

12 Wyszecki, G. and Stiles, W. S. Color Science: Concepts and Methods, Quantitative Data and Formulae, 2nd edition, 1982, Table I (5.5.6), pp. 817-821 (John Wiley, Chichester).

$13 \mathrm{Yu}$, D. K. C. and Oulton, D. P. Colour difference acceptability for calibrated digital images. Digital Futures 2000, University of Westminster, 11-13 September 2000; in Imag. Sci. J., 2000, 48, 165-176.

14 Berns, R. S. A generic approach to color modeling. Col. Res. Appl., 1997, 22(5), 318-325.

15 Oulton, D. P. The properties of multiple CMF determinations using alternative primary sets part I: evidence and modeling. Col. Res. Appl., 2004, 29, 273-284.

16 Sproson, W. N. Colour Science in Television and Display Systems, 1983 (Adam Hilger, Bristol).

17 Stokman, H. G., Gevers, T. and Koenderink, J. J. Color measurement by imaging spectrometry. Comput. Vision Image Understand., 2000, 79, 236-249 (on-line at www.idealibrary.com).

18 Taylor, H. 'The presence and effects of observer metamerism', BSc Hons. Dissertation, Department of Textiles, UMIST, 1999. 